\title{
EFFECTS OF DIFFERENT DEVELOPMENT STAGES AND STRESS GRADIENT ON DENSITY-BIOMASS EXPONENT AND SIZE INEQUALITY OF HALOXYLON AMMODENDRON (C.A. MEY)
}

\author{
ZHAO, Z. L. - HU, X. J. - SONG, Y. Y.* \\ Department of Forestry, Agricultural College, Xinjiang Shihezi University \\ Road of North 4th, Shihezi City, Xinjiang 832003, China \\ *Corresponding author \\ e-mail:syy.agr.shzu@126.com
}

(Received 10 $0^{\text {th }}$ Jun 2019; accepted $11^{\text {th }}$ Oct 2019)

\begin{abstract}
Quantifying the biomass-density (M-N) exponent and size inequality in plant communities has become a long-standing issue in both theoretical and empirical studies. The biomass-density $(\mathrm{M}-\mathrm{N})$ exponent and size inequality of the tree populations have not been studied so widely as that of herbaceous plants. we studied the variation of biomass-density $(\mathrm{M}-\mathrm{N})$ exponent and size inequality at different stages of development clusters along erosion gradient in Haloxylon ammodendron populations. The results showed that the value of the M-N exponent ranged from 1.21 to 1.73 in sapling stages, -0.691 to 0.437 in Adult stages and -0.934 to -0.812 in senescence phase along a erosion gradient, the M-N exponents at different stages of development showed an downward trend, and indicated that when the density of the cluster increases, which is a process of self-thinning. Moreover, the populations size inequality in different locations decreased with the increase of wind erosion intensity. The size inequality also showed a downward trend in various stages of development, that of the first two stages of development were significantly greater than the later development stages. Our study showed that interactions among individuals at different stages of development have an important impact on the population structure.
\end{abstract}

Keywords: density, interaction, Haloxylon ammodendron, Severe environment, cluster

\section{Introduction}

Mass-density $(\mathrm{M}-\mathrm{N})$ scaling relationship describes variation in population density with body size in the plant community and can be described as $M=K \times N^{\gamma}$. In past 40 years, many ecological scientists have devoted themselves to research $\mathrm{M}-\mathrm{N}$ scaling relationship, and most of these researches proved the $-3 / 2$ (Yoda et al., 1963) or $-4 / 3 \mathrm{M}-$ $\mathrm{N}$ scaling relationship (Equist et al., 1998; Brian et al., 2003). However, $-3 / 2$ and $-4 / 3$ exponents of $\mathrm{M}-\mathrm{N}$ scaling relationship were also criticized on theoretical and empirical grounds (Kozlowski and Konarzewski, 2004; Deng et al., 2006), controversy continues over the underlying value and variability of the $\mathrm{M}-\mathrm{N}$ scaling exponent. Many literatures indicate that $\gamma$ varies with soil fertility (Morris, 2003), water availability (Deng et al., 2006), aridity gradient (Bai et al., 2010), hormone response (Zhang et al., 2006, 2016) and salinity gradient (Zhang et al., 2010), all of the above experiments showed $\gamma$ is an exponent that increases as the pressure gradient increases or vary with different forest types (Zhang et al., 2011). Chu found that the density regulation of plant populations is actually affected by the positive and negative effects among individuals (Chu et al., 2008), which think that environmental factors can affect the law of density regulation by affecting the interaction between plants. The law of density regulation is the external performance of interaction between plants and plants, between plants and the 
environment. The result of plant competition leads to a decrease in the growth rate of the individual, which in turn affects biomass accumulation and ultimately affects the biomass-density relationship. If the interaction between individuals is not competition, but reciprocity, it is inevitable that the average individual biomass will increase with the increase of density (Chu et al., 2008; Lin et al., 2012). Therefore, it can be assumed that the interaction among individual plants and density regulation exponent is closely related.

A lot of studies have shown that the balance of competition and facilitation shifts among the various life stages of two interacting species (Chapin et al., 1994; Long et al., 2014; Zhang et al., 2012; Lin et al., 2012). However, facilitative and competitive mechanisms do not act in isolation from each other in nature, and by co-occurring within the same community, and even between the same individuals, they may produce complex and variable effects. Some studies have shown that life stages (e.g. seed, seedling, juvenile, pre-reproductive adult, reproductive adult, senescent adult) may affect the outcome of interactions between plant species (Rousset et al., 2000). Since the positive and negative interactions are different at different stages of development, is the M-N scaling exponent different? Or how does it change in different stages of development? It seems to attract little attention.

Large variation in individual size is a ubiquitous feature of natural plant populations and this variation has major implications for plant ecology and evolution. There have been a few studies on size inequality among individuals (Weiner et al., 2001; Chu et al., 2009). They found that the different spatial patterns, different density gradients of plant populations (Weiner et al., 2001) and different pressure gradients (Zhang et al., 2012) have a significant impact on population size inequality, Weiner (Weiner et al., 2001) showed that at low densities and early in growth, spatial pattern played a more important role than size asymmetry of competition in generating size inequality, but at high densities or after longer periods of growth, the size asymmetry of competition was more important than spatial pattern. Zhang et al.'s (2012) experiments on the growth of mung bean with different intensity of ultraviolet radiation found that the balance among positive interactions, stress, and mortality driven by competition determined the size inequality of the populations (Zhang et al., 2012). However, studies on the variation of size inequality in developmental stages of tree populations are rare.

Haloxylon ammodendron, which belongs to genus Haloxylon, is a typical desert plant, primarily found on shifting or semi-shifting sand dunes of the narrow sub-desert areas in middle and western Asia (Zou et al., 2010). The species is also termed a super xerophyte due to its particular drought tolerance that enables it to survive in prohibitive environments (Gao et al., 2010). This species had been selected as a pioneer plant for sand dune stabilization, and it is an excellent plant for evaluating disturbance and vegetation restoration in Gurbantunggut Desert (Bedunah and Schmidt, 2000). However, the area of natural Haloxylon ammodendron forest is decreasing because of land development, groundwater mining, excessive deforestation, and overgrazing. Hence, Haloxylon ammodendron has been listed as a national grade 3 endangered plant in China (Guo et al., 2005). A 35-yr-old replacement series of Haloxylon ammodendron clusters in the Gurbantunggut Desert has provided a condition to study the density change of cluster under severe stress environments. The Haloxylon ammodendron is distributed in the form of cluster on top of sand dunes in the desert, which suffer from strong wind erosion and they grows in aggregate pattern. There is no more aggregation pattern to be found except at the top of the hill. Interestingly, there is a significant phase 


$$
-14667 \text { - }
$$

difference in the density change process of Haloxylon ammodendron clusters, therefore, it provides us with a convenient way to study the variation of the $\mathrm{M}-\mathrm{N}$ scaling exponent and changes in size inequality in different developmental stages. Thus we will ask the following three questions: (1) How does the $\mathrm{M}-\mathrm{N}$ exponent change in different developmental stages of Haloxylon ammodendron? (2) How does the size inequality of Haloxylon ammodendron change during different developmental stages? (3) Is there a positive self-thinning exponent and how much is its size?

\section{Materials and methods}

\section{Description of the study site}

This study has been conducted along a natural wind speed gradient at five sites (Xinhu, Shihezi, Kuitun, Guertu and Jinghe) in the Gurbantunggut Desert, Xinjiang, which is located in the hinterland of the Junggar Basin in northwest China ( $44^{\circ} 11^{\prime}$ to $46^{\circ} 20^{\prime} \mathrm{N}, 84^{\circ} 31^{\prime}$ to $90^{\circ} 00^{\prime} \mathrm{E}$ ), with an area of up to $48,800 \mathrm{~km}^{2}$. The Gurbantunggut Desert is the second largest desert in China. Due to its long distance from any ocean, it has a temperate arid desert climate. The annual precipitation is $80 \mathrm{~mm}$ to $190 \mathrm{~mm}$. The annual average temperature is 5.0 to $5.7^{\circ} \mathrm{C}$. Haloxylon ammodendron is the dominant species in this desert, and the ephemeral plant layer is well-developed (Figs. 1 and 2).

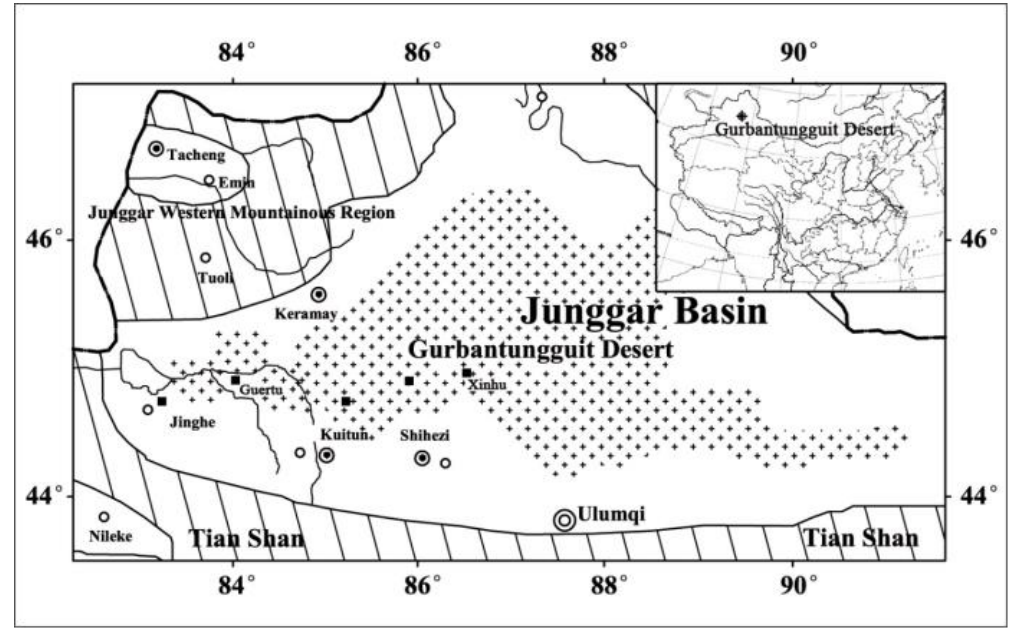

Figure 1. The maps of different sampling sites
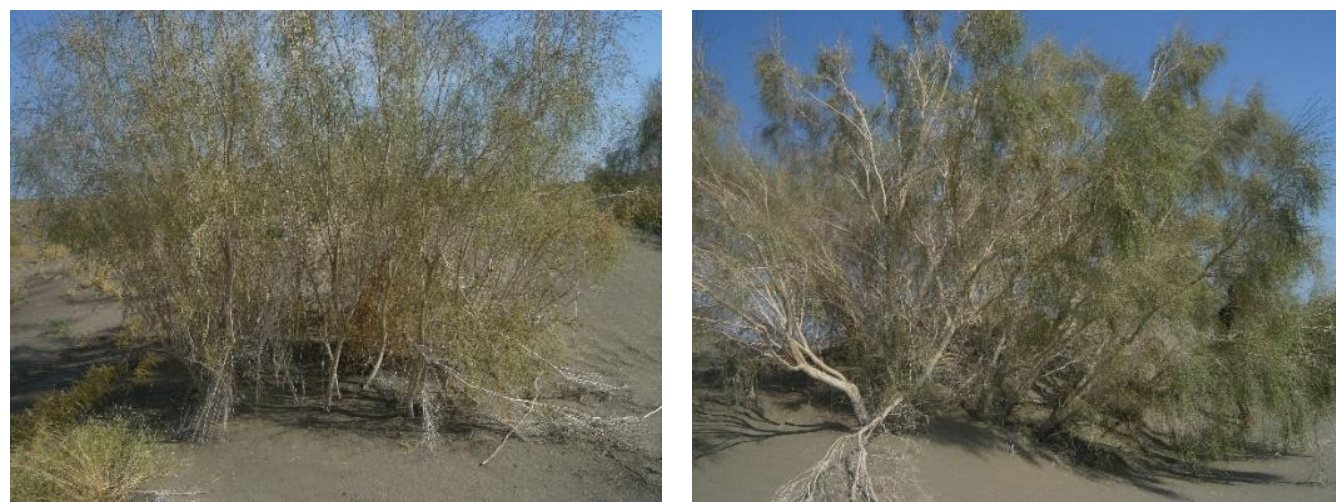

Figure 2. Research sites and species 


\section{Field surveying}

At the beginning of 2016, we chose five sites at the study area, and all the sites were located in different spot from low wind speed to high wind speed (Table 1), since the difference in height and shape of sand dunes at each location is not large, the influence of different shapes on wind speed can be ignored, in addition, the rainfall in the entire Gurbantunggut desert is almost the same. Data were collected from local weather stations respectively. A $40 \times 40 \mathrm{~m}$ plot was selected at different research sites and the clusters of five development stages were selected for each plot, to avoid sample size inhomogeneity, we initially selected 10-15 clusters for each stage of development, then selected three representative clusters from among them. According to the distribution situations of the age of Haloxylon ammodendron clusters, we only need to find the maximum age for each cluster because each Haloxylon ammodendum cluster is composed of uneven-aged individuals. Haloxylon ammodendron is divided into five developmental stages, which can be expressed as follows, sapling: 1-6 yr; Adult phase I: 7-13 yr; Adult phase II: 14-20 yr; senescence phase: 21-27 yr; death phase: 28-34 yr; Age formula comes from previous research results (Song et al., 2011), and they are used to determine the age of the cluster. In addition, we selected $1 \times 1 \mathrm{~m}$ quadrat in each cluster to collect the basal diameter, crown width and tree height data of each Haloxylon ammodendum. Moreover, in order to reduce difference of sample scale and avoid edge effects, each plot is close to the middle of the clusters and each quadrat was replicated three times, meanwhile, the number of Haloxylon ammodendron per square meter was measured to calculate the density.

Table 1. Descriptions of the five experimental sites

\begin{tabular}{c|c|c|c|c|c}
\hline \multirow{2}{*}{ Parameters } & \multicolumn{5}{c}{ Site } \\
\cline { 2 - 6 } & Xinhu & Shihezi & Kuitun & Guertu & Jinghe \\
\hline Latitude & $45^{\circ} 13$ & $45^{\circ} 26^{\prime}$ & $44^{\circ} 89^{\prime}$ & $45^{\circ} 41$ & $44^{\circ} 75^{\prime}$ \\
Longitude & $86^{\circ} 55$ & $85^{\circ} 88^{\prime}$ & $85^{\circ} 47^{\prime}$ & $84^{\circ} 26$ & $83^{\circ} 35^{\prime}$ \\
Altitude $(\mathrm{m})$ & 454 & 443 & 460 & 442 & 436 \\
Annual mean temperature $\left({ }^{\circ} \mathrm{C}\right)$ & 5.2 & 5.1 & 5.5 & 5.6 & 5.4 \\
Annual mean precipitation $(\mathrm{mm})$ & 230 & 210 & 182 & 173 & 144 \\
Annual mean potential evaporation $(\mathrm{mm})$ & 1280 & 1300 & 1810.7 & 1960 & 2000 \\
Wind speed (m/s) & 1.9 & 2.1 & 2.8 & 3.2 & 3.7 \\
Spring mean wind speed (m/s) & 4 & 5.4 & 6.5 & 7 & 9.5 \\
\hline
\end{tabular}

The above data are from the local monitoring result from 1975 to 2015

\section{Biomass estimation}

To picks representative Haloxylon ammodendron individuals of each age to measure his above-ground biomass, height, basal diameter for deriving the biomass calculation formula, three representative Haloxylon ammodendron plants were selected for each age, the whole tree (above the ground) was excavated and weighed, and adopt the age separation excavation method, a total of 45 Haloxylon ammodendron plants were dug at each site. Parts of the samples were brought back to the laboratory to be dried and weighed, and then above ground biomass (ATB) was calculated accordingly. The biomass calculation formulas of Xinhu, Shihezi, Kuitun, Guertu and Jinghe are respectively: 


$$
\begin{aligned}
\mathrm{B}_{\text {Xin }} & =0.2012\left(d^{2} h\right)^{0.8231} \\
\mathrm{~B}_{\text {Shi }} & =0.1965\left(d^{2} h\right)^{0.7953} \\
\mathrm{~B}_{\text {Kui }} & =0.1834\left(d^{2} h\right)^{0.7826} \\
\mathrm{~B}_{G u} & =0.1238\left(d^{2} h\right)^{0.7492} \\
\mathrm{~B}_{\text {Jing }} & =0.1107\left(d^{2} h\right)^{0.7223}
\end{aligned}
$$

where $\mathrm{B}$ were the individual biomass weight, $\mathrm{d}$ were basal diameter, $\mathrm{h}$ were tree height.

\section{Calculation of the size inequality of individual Haloxylon ammodendron}

We analysed the size inequality of different parameters which can be expressed by the coefficient of variation (CV) of basal diameter, height, individual mass of Haloxylon ammodendron. To quantify size inequality in Haloxylon ammodendron clusters, the CV used is $\mathrm{CV}=\mathrm{SD} / X_{\text {mean }}$ (Weiner et al., 2001; Chu et al., 2009), SD is the standard deviation, $X_{\text {mean }}$ is the sample mean.

\section{Collection of stress factors}

The collected field data are supposed to contain the five different wind erosion intensity which are to be used as abiotic stress factors, usually from March to May, the average wind speeds of Xinhu, Shihezi, Kuitun, Guertu and Jinghe are $4 \mathrm{~m} / \mathrm{s}, 5.4 \mathrm{~m} / \mathrm{s}$, $6.5 \mathrm{~m} / \mathrm{s}, 7 \mathrm{~m} / \mathrm{s}, 9.5 \mathrm{~m} / \mathrm{s}$ respectively, which show a trend of decreasing from west to east. Wind speed at the top of the dune is greater than that of the sand-driving wind velocity $(6 \mathrm{~m} / \mathrm{s})$ and the top of the sand hill is exposed to the process of wind erosion, the above data are from the local monitoring result from 1975 to 2015.

\section{Data analysis}

All data were analyzed by spss 22 and Excel 2016, the plotted results was conducted via Origin 8.0 software, the scaling exponents and intercepts of $\mathrm{M}-\mathrm{N}$ relationships were estimated by the standard major axis (SMA) regression of log-transformed data. Significance between the CV value (coefficient of variation) of different stages of development are expressed by the means of the Duncan's test, $p>0.05$.

\section{Results}

\section{Density changes}

For the trend of the density changes of Haloxylon ammodendron clusters in five sites with wind erosion, a rapidly increased is found firstly in Sapling phase while a rapid decrease is found in Adult phase I, after that, a slow decline trend appears (Fig. 3). In Xinhu, Haloxylon ammodendron reaches its maximum density $\left(30 \mathrm{trees} / \mathrm{m}^{2}\right)$ at the ninth age, subsequently, the density is in rapid decline from age 9 to age 13, after that the decline in density enters a relatively slow process. The Kuitun site reached its maximum density $\left(41 \mathrm{trees} / \mathrm{m}^{2}\right)$ in the seventh year. Seven to eleventh years is regarded as a rapid decline period, which is slightly steeper than that Shihezi and Xinhu (Fig. 3). In Jinghe, 
the density reach its maximum $\left(60\right.$ trees $\left./ \mathrm{m}^{2}\right)$ in the fourth year. Similarly, in the period from fourth to eighth year, a rapid decline period can be seen, this stage in Jinghe has the steepest change compared with the change other four location. After that the downward trend began to be slower (Fig. 3). Compared to the other four locations at the same time, obviously, the density of Haloxylon ammodendron increased with the increase of wind erosion and showed a more intense pattern of aggregation. Another fact is that wind erosion significantly accelerated the onset of Haloxylon ammodendron density-dependent mortality, especially at the Jinghe site, the density-dependent mortality occurred earlier than that in other four locations. However, the density of the five sites during the death phase is similar (Fig. 3).

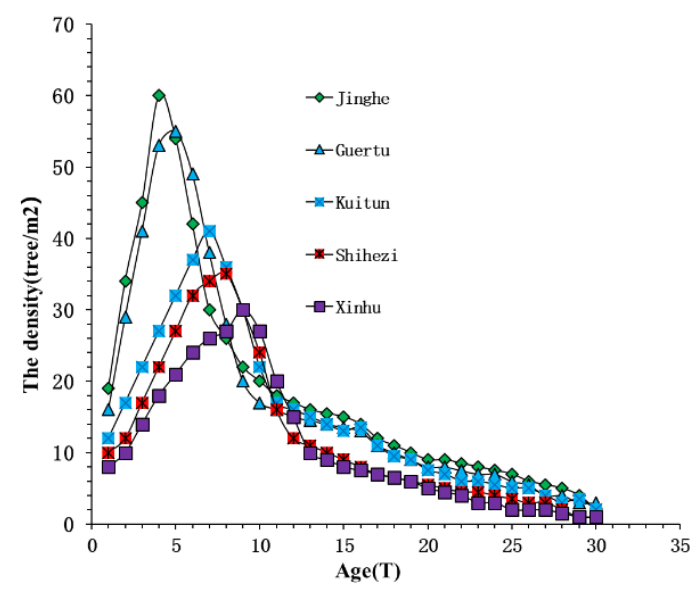

Figure 3. The trend of the density change of Haloxylon ammodendron at five different locations, respectively

\section{The M-N exponents}

There are three parts of density changes concerning rise and rapid fall, slow decline during self-thinning period (Fig. 4), so we calculate the M-N exponents in three sections respectively. The value of the M-N exponent ranged from 1.73 to 1.21 for phase along a erosion gradient (Table 2), the density exponent of the sapling phase were significantly larger than the traditional $-3 / 2$ and $-4 / 3(p>0.05)$ based on the $95 \% \mathrm{CI}$; the density exponent of the adult phase were also significantly different from $-3 / 2$ and $-4 / 3$. In the senescence phase, the density exponent of Xinhu, Kuitun and Jinghe had no significant difference with $-4 / 3$, which indicates that the relationship between the average individual biomass and density on the ground during the senescence phase follows the 4/3 M-N exponent rule, meanwhile, in the same sites, with the increase of Haloxylon ammodendron age, the M-N exponents at different stages of development showed a downward trend.

\section{Size inequality}

At the top of the dune, different wind speed significantly influenced the size inequality of the Haloxylon ammodendron different parameters. For the basal diameter, High, Crown width and biomass, the $\mathrm{CV}$ value (coefficient of variation) of size inequality at Jinghe (strong wind) was lower than those in other four places, the highest $\mathrm{CV}$ value (coefficient of variation) is found at Xinhu site (Fig. 5). The CV Values 
(coefficient of variation) of various parts under different wind erosion conditions in the same developmental stage are also significantly different (Fig. 5), The CV values (coefficient of variation) of size inequality of the sapling stage and Adult phase I was significantly higher than those in other stages of development.

Table 2. Self-thinning exponents ( $\gamma)$ and intercepts (K) for above-ground across different developmental stages as estimated by SMA regression of log-transformed data

\begin{tabular}{c|c|c|c|c|c|c|c|c}
\hline $\begin{array}{c}\text { Developmental } \\
\text { stage }\end{array}$ & Sites & $\begin{array}{c}\text { Regression } \\
\text { relationship }\end{array}$ & Slope $(\gamma)$ & $\mathbf{9 5 \%}$ CI & Intercept(k) & $\mathbf{9 5 \%}$ CI & $\mathbf{R}^{2}$ & n \\
\hline \multirow{5}{*}{ Sapling phase } & Xi hu & M:D & 1.73 & $1.34,2.24$ & -0.1083 & $-0.735,0.376$ & 0.932 & 8 \\
& Shi he zi & M:D & 1.94 & $1.34,2.81$ & -0.606 & $-1.7,0.1528$ & 0.926 & 6 \\
& Kui tun & M:D & 1.73 & $1.54,2.15$ & -0.19 & $. .-1.27,-0.411$ & 0.968 & 5 \\
& Guertu & M:D & 1.27 & $0.992,1.62$ & -0.609 & $-1.13,-0.199$ & 0.994 & 4 \\
& Jinghe & M:D & 1.21 & $0.93,1.57$ & -0.712 & $-1.27,-0.281$ & 0.996 & 4 \\
\hline & Xi hu & M:D & -0.691 & $-1.031,-0.463$ & 3.5 & $3.21,3.93$ & 0.95 & 5 \\
& Shi he zi & M:D & -0.739 & $-1.16,-0.469$ & 3.38 & $3.12,4.04$ & 0.935 & 5 \\
Adult phase & Kui tun & M:D & -0.467 & $-0.774,-0.282$ & 2.82 & $2.55,3.26$ & 0.918 & 5 \\
& Guertu & M:D & -0.422 & $-0.559,-0.318$ & 2.44 & $2.28,2.69$ & 0.976 & 5 \\
& Jinghe & M:D & -0.437 & $-1.015,-0.1883$ & 2.43 & $2.04,3.36$ & 0.735 & 5 \\
\hline \multirow{5}{*}{$\begin{array}{c}\text { Senescence } \\
\text { phase }\end{array}$} & Xi hu & M:D & -0.934 & $-1.09,-0.801$ & 3.98 & $3.9,4.08$ & 0.922 & 17 \\
& Shi he zi & M:D & -0.782 & $-0.901,-0.679$ & 3.78 & $3.72,3.84$ & 0.918 & 20 \\
& Kui tun & M:D & -1.021 & $-1.18,-0.881$ & 3.95 & $3.84,4.08$ & 0.905 & 21 \\
& Guertu & M:D & -1.1 & $-1.31,-0.925$ & 3.74 & $3.59,3.92$ & 0.858 & 22 \\
& Jinghe & M:D & -0.812 & $-1,-0.659$ & 3.5 & $3.35,3.68$ & 0.798 & 22 \\
\hline
\end{tabular}

$\mathrm{M}$ is aboveground average individual biomass, $\mathrm{N}$ is density. $\mathrm{M}: \mathrm{N}$ is $\log \mathrm{M}: \log \mathrm{N} .95 \%$ CIs are confidence intervals for $\lambda$ and $\mathrm{K}$, $\mathrm{n}$ is the number of samples

\section{Discussion}

\section{The density changes with developmental stages}

Our natural Haloxylon ammodendron clusters show there is a pattern of humpshaped density change, apparently, Haloxylon ammodendron cluster density in the Sapling phase showed a rapid growth trend. The survival rate of clusters was significantly higher than the mortality rate when the maximum density is reached, but due to the fact that individual clusters are too dense, some smaller individuals do not receive sufficient light and other nutrition so that the mortality rate has increased dramatically, resulting in a large number of individual deaths during the adult I period. Experience observation suggests that light is an important factor in the growth of Haloxylon ammodendron, subsequently, the trend of decreasing density has gradually become more moderate. Moreover, the worse the environmental gradient is, the sooner the Haloxylon ammodendron cluster enters self-thinning period (Fig. 3). This phenomenon was inconsistent with the previous model study in which the asymmetric facilitation delays the onset of density-dependent mortality (Lin et al., 2015), this situation may be caused by the fact that the cluster is trying to resist wind erosion, a large number of individuals colonize the top of the dune in high density, resulting in density-dependent deaths. The initial density is proportionally related to the environmental stress gradient (Fig. 3), in order to compete limited resources, the higher the initial density is, the more fierce the density-dependent death among individual will be. Moreover, the density decreasing degree in Adult phase I is significantly different from that of subsequent periods. So we speculate that the essential cause of phase 
change of the density of cluster during different development period is the result of the transformation of the interactions between individuals (Chu et al., 2008). Therefore, it is very meaningful to study the characteristics of density variation between different developmental stages of tree populations under extreme conditions. How to changes the interaction among Haloxylon ammodendron individuals with the density and development stage will be an important direction for future study.
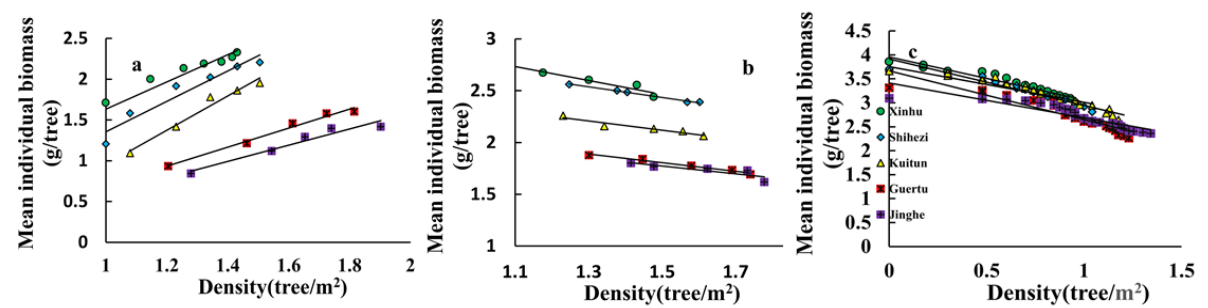

Figure 4. Density-biomass exponents along wind erosion gradient at different development stages. The self-thinning line of sapling phase (a), the self-thinning line of adult phase I $(b)$, the self-thinning line of adult (c)

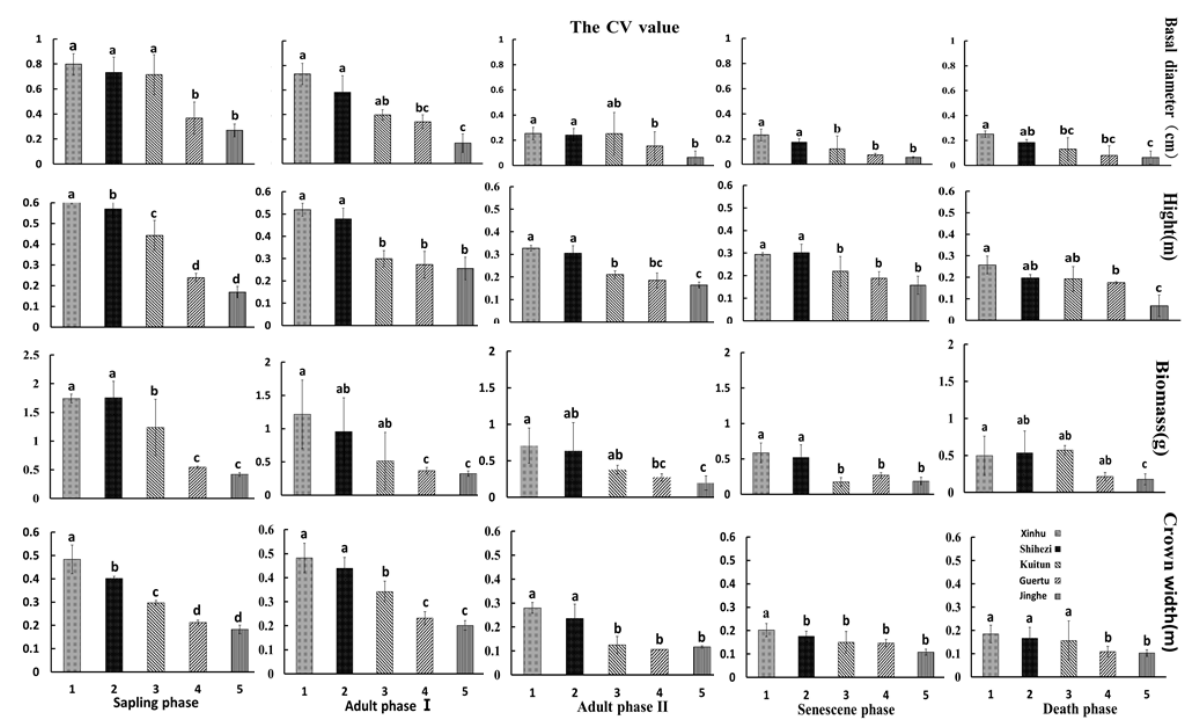

Figure 5. The CV value of the stem, High, Crown width and the whole Haloxylon ammodendron biomass were calculated under different wind erosion stress gradient in the different stages of growth. Different places represent different wind erosion. 95\% confidence intervals for unbiased values were determined by using the Duncan's test

\section{The M-N (self-thinning) exponents and size inequality}

In our study, there was no general predicted value that can describe all the $\mathrm{M}-\mathrm{N}$ relationships exponents for different stages development across different wind erosion gradient, also different from the $-3 / 2$ and $-4 / 3$ "law" (Deng et al., 2006; Zhang et al., 2011, 2017). According to the definition of population self-thinning, when the mortality rate within the population reaches $20 \%$, it indicates that density dependent death has occurred (Maina et al., 2015). The Haloxylon ammodendron cluster we studied was originally planted on top of a dune in the form of a small area, in order to resist the 
erosion of wind, the density of Haloxylon ammodendron seedlings reaches dozens, so that the intertwined roots fix the sand in a higher density, as the Haloxylon ammodendron cluster grows, the clusters expand radially around the center of the initial colonization site and grow usually in high density and seeds added in subsequent years, except for falling inside the clusters, must fall near the initial settlement, and if they are far away, they will not survive. So when the clusters grow to 2 to 3 years, the competition among individuals is more intense, a lot of individuals begin to die and can beyond $25 \%$ mortality rate (Table 3 ), at the same time, the occurrence of this process is accompanied by a higher survival rate and the survival rate is much greater than the mortality rate, therefore, the essential reason for the increase in density is that the new planting individual counteracts the death individual, resulting in a "trend of increasing density". This phenomenon is different from the tree population self-thinning in the traditional sense that is generally colonized in a certain area in a random distribution, the occurrence of self-thinning is often accompanied by a decrease in population density (Xu et al., 2018; Zhang et al., 2011; Deng et al., 2006; Chu et al., 2010). Moreover, although a small number of individuals die in the early stages of population development, but the death is not density-dependent death, but is caused by other external causes, such as disease, weakness or harsh environmental conditions, so selfthinning can be a process of increasing density, which proves that there is a positive self-thinning exponents in nature. The self-thinning exponent of three-phase decreases monotonously as the different developmental stages increases (Fig. 4). Self-thinning exponent was positive in the previous one to five years, after the cluster reaches the highest density, self-thinning has become very fierce, especially in Adult phase I. Later it shows a negative Self-thinning exponent, which may be caused by the fact that there is strong wind erosion stress at the top of dune, thus there may be a strongly facilitation effects among Haloxylon ammodendron individuals (Bertness et al., 1994; Callaway and Walker et al., 1997; Callaway et al., 2002, 2007). The strong positive effect promotes the individual average biomass of cluster to increase with the density in Sapling phase. In fact, this situation has been predicted by previous research, which suggested that the allometric exponents (or regression slopes) might even become positive under the most extreme conditions where facilitation is commonly observed, the above study predicted this result, but it was not confirmed in reality yet (Deng et al., 2006). The results of this paper has just verified the existence of positive M-N exponents and proved the complexity of the self-thinning patterns of different species. It can be seen herbs have a shorter developmental history, but not long enough for us to observe its phase effects. As for arbor plants with long developmental history, it is more convenient to observe a series of change characteristics during the developmental stage. Therefore, it is very meaningful to discover the characteristics of different interacting species and natural stress factors (Maestre et al., 2009).

In addition, the coefficient of variation of different parameters of Haloxylon ammodendron at different developmental stages also decreases with increasing wind erosion in this same location, however, previous study (Zhang et al., 2012) showed that size inequality (coefficient of variation) was simply monotonic decreases in Mung beans growing phase 1 with the increase of UV-B stress strength, but non-monotonic decreases is with UV-B radiation increased in phase 2 and 3 . They attribute the result to the spatial pattern of plant growth and the symmetry of competition (Chu et al., 2009; Weiner et al., 2001). Our study does not need to consider these factors, because the entire process of cluster development is in a state of self-thinning, so there is no effect 
of spatial patterns. It can more accurately reflect with environmental pressure gradient how the size inequality (coefficient of variation) changes for each developmental stage. For each parameter, sapling, We speculate, this may be related to the predominance of facilitation in the previous period, in the same facilitation patterns sapling stage and adult phase I, the clusters are constantly recruit new individuals and accompanied by the death of some individuals. Haloxylon ammodendron individuals that are constantly settled and death exacerbate the size inequality of clusters, lead to a relatively high size variation, in the adult phase II, senescene phase, death phase the changes in density have slowed and subsequent supplements can only survive for 1-3 years. The difference in the age of surviving individuals at this stage is much smaller than that in the previous period, thus clusters show a more neat trend.

Table 3. Changes of Haloxylon ammodendron clusters plants number during sapling phase

\begin{tabular}{|c|c|c|c|c|c|c|}
\hline Age & & Jinghe & Guertu & Kuitun & Shihezi & Xinhu \\
\hline \multirow{3}{*}{ One year } & Survival & 19 & 16 & 12 & 10 & 8 \\
\hline & Deaths/current year & 0 & 0 & 0 & 0 & 0 \\
\hline & Mortality rate & 0 & 0 & 0 & 0 & 0 \\
\hline \multirow{3}{*}{ Two year } & Survival & 34 & 29 & 17 & 12 & 10 \\
\hline & Deaths/current year & $12(9)$ & $10(8)$ & $8(5)$ & $6(4)$ & $4(3)$ \\
\hline & Mortality rate & $26 \%$ & $25 \%$ & $32 \%$ & $33 \%$ & $28 \%$ \\
\hline \multirow{3}{*}{ Three year } & Survival & 45 & 41 & 22 & 17 & 14 \\
\hline & Deaths/current year & $34(15)$ & $26(11)$ & $16(6)$ & $14(5)$ & $12(5)$ \\
\hline & Mortality rate & $33 \%$ & $28 \%$ & $26 \%$ & $32 \%$ & $36 \%$ \\
\hline \multirow{3}{*}{ Four year } & Survival & 60 & 53 & 27 & 22 & 18 \\
\hline & Deaths/current year & $60(18)$ & $56(13)$ & $31(10)$ & $23(8)$ & $18(6)$ \\
\hline & Mortality rate & $30 \%$ & $25 \%$ & $37 \%$ & $35 \%$ & $33 \%$ \\
\hline \multirow{3}{*}{ Five year } & Survival & 54 & 55 & 32 & 27 & 21 \\
\hline & Deaths/current year & $98(22)$ & $83(18)$ & 35 (19) & $43(8)$ & $29(7)$ \\
\hline & Mortality rate & $41 \%$ & $33 \%$ & $60 \%$ & $29 \%$ & $34 \%$ \\
\hline \multirow{3}{*}{ Six year } & Survival & 42 & 49 & 37 & 32 & 24 \\
\hline & Deaths/current year & $107(14)$ & $100(14)$ & $77(14)$ & $67(14)$ & $40(7)$ \\
\hline & Mortality rate & $35 \%$ & $29 \%$ & $37 \%$ & $43 \%$ & $31 \%$ \\
\hline
\end{tabular}

The survival number and death number in the table are cumulative values, and all death individuals will disappear due to wind erosion two to three years after death. Current Year is the number of deaths is the number of individuals who died in the current year. When calculating the mortality rate, the number of deaths is the number of individuals who died in the current year

\section{Conclusion}

(1) The density of clusters increased rapidly at the sapling stage, decreased rapidly at the adult stage, and then decreased slowly at the later stage. By comparing five different wind erosion sites, we can clearly find that the density of Haloxylon ammodendron increases with the increase of wind erosion intensity, showing a denser growth pattern. Wind erosion significantly accelerated density-dependent death of Haloxylon ammodendron.

(2) the self-thinning indexes of Haloxylon ammodendron clusters along different wind erosion gradients were all positive at the rapid rising stage, it is significantly 
different from the traditional $-3 / 2$ and $-4 / 3$ self-thinning indices; in the stage of rapid density decline, the negative value presents an upward trend along the wind erosion gradient, which is significantly different from the traditional $-3 / 2$ and $-4 / 3$ self-thinning indexes; the difference between the $-4 / 3$ self-thinning index and self-thinning index of Xinhu, Kuitun and Jinghe in the stages of aging and death is not obvious, indicating that the self-thinning stage conforms to the $-4 / 3$ self-thinning rule. With the growth and development of cluster, the self-thinning index of the same location showed a decreasing trend.

(3) The CV values of the cluster size inequality of the sapling stage and adult phase I was significantly higher than those in other stages of development.

Future research should explicitly evaluate how to incorporate different developmental stages of arbor species into current conceptual and mathematical models, which can be used to improve the SGH (stress-gradient hypothesis) framework. The computer models of tree species need to be made to explore the changing patterns of positive and negative interactions among individuals, the facilitative effect from key species is essential for ecological system diversity and stability (Brooker et al., 2008; Vellend, 2008), which help us extend the SGH (stress-gradient hypothesis) framework and foster research on facilitation in ecology (Brooker et al., 2005, 2008; Maestre et al., 2009).

\section{REFERENCES}

[1] Bai, Y., Zhang, W., Jia, X., et al. (2010): Variation in root: shoot ratios induced the differences between above and belowground mass-density relationships along an aridity gradient. - Acta Oecologica 36(4): 393-395.

[2] Bertness, M. D., Callaway, R. (1994): Positive interactions in communities. - Trends in Ecology \& Evolution 9(5): 191-193.

[3] Brian, J. E., Evan, P. E., Travis, E. H., Huxman, T. E., Allen, A. P., Ignace, D. D., Gillooly, J. F. (2003): Scaling metabolism from organisms to ecosystems. - Nature 423: 639-642.

[4] Brooker, R., Kikvidze, Z., Pugnaire, F. I., et al. (2005): The importance of importance. Oikos 109(1): 63-70.

[5] Brooker, R. W., Maestre, F. T., Callaway, R. M., et al. (2008): Facilitation in plant communities: the past, the present, and the future. - Journal of Ecology 96(1): 18-34.

[6] Callaway, R. M. (2007): Positive Interactions and Interdependence in Plant Communities. - Springer, Dordrecht.

[7] Callaway, R. M., Walker, L. R. (1997): Competition and facilitation: a synthetic approach to interactions in plant communities. - Ecology 78(7): 1958-1965.

[8] Callaway, R. M., Brooker, R. W., Choler, P., et al. (2002): Positive interactions among alpine plants increase with stress. - Nature 417(6891): 844-848.

[9] Chapin, F. S., Walker, L. R., Sharman, F. L. C. (1994): Mechanisms of primary succession following deglaciation at Glacier Bay, Alaska. - Ecological Monographs 64(2): 149-175.

[10] Chu, C. J., Maestre, F. T., Xiao, S., et al. (2008): Balance between facilitation and resource competition determines biomass-density relationships in plant populations. Ecology letters 11(11): 1189-1197.

[11] Chu, C. J., Weiner, J., Maestre, F. T., et al. (2009): Positive interactions can increase size inequality in plant populations. - Journal of Ecology 97(6): 1401-1407. 
[12] Chu, C. J., Weiner, J., Maestre, F. T., et al. (2010): Effects of positive interactions, size symmetry of competition and abiotic stress on self-thinning in simulated plant populations. - Annals of Botany 106(4): 647-652.

[13] Deng, J., Wang, G., Morris, E., et al. (2006): Plant mass-density relationship along a moisture gradient in north-west China. - Journal of Ecology 94(5): 953-958.

[14] Gao, S., Su, P. X., Yan, Q. D., et al. (2010): Canopy and leaf gas exchange of Haloxylon ammodendron under different soil moisture regimes. - Science China Life Sciences 53(6): 718-728.

[15] Koz Owski, J., Konarzewski, M. (2004): Is West, Brown and Enquist's model of allometric scaling mathematically correct and biologically relevant? - Functional Ecology 18(2): 283-289.

[16] Lin, Y., Berger, U., Grimm, V., et al. (2012): Differences between symmetric and asymmetric facilitation matter: exploring the interplay between modes of positive and negative plant interactions. - Journal of Ecology 100(6): 10.

[17] Long, Y., Zhang, J., Tian, X., et al. (2014): De novo assembly of the desert tree Haloxylon ammodendron (C. A. Mey.) based on RNA-Seq data provides insight into drought response, gene discovery and marker identification. - BMC Genomics 15(1): 1111.

[18] Maestre, F. T., Callaway, R. M., Valladares, F., et al. (2009): Refining the stress-gradient hypothesis for competition and facilitation in plant communities. - Journal of Ecology 97(2): 199-205.

[19] Maina, J. N., Wang, Q. (2015): Seasonal response of chlorophyll a/b ratio to stress in a typical desert species: Haloxylon ammodendron. - Arid Land Research and Management 29(3): 321-334.

[20] Morris, E. C. (2003): How does fertility of the substrate affect intraspecific competition? Evidence and synthesis from self-thinning. - Ecological Research 18(3): 287-305.

[21] Rousset, O., Lepart, J. (2000): Positive and negative interactions at different life stages of a colonizing species (Quercus humilis). - Journal of Ecology 88(3): 401-412.

[22] Song, Y. Y., Chu, G. M., Xiao-Jing, H. U. (2011): Relationship of diameter-class and age-class of Haloxylon ammodendron population in Gurbantonggut Desert. - Acta Botanica Boreali - Occidentalia Sinica 31(4): 808-814.

[23] Vellend, M. (2008): Effects of diversity on diversity: consequences of competition and facilitation. - Oikos 117(7): 1075-1085.

[24] Weiner, J., Stoll, P., Mullerlandau, H., et al. (2001): The effects of density, spatial pattern, and competitive symmetry on size variation in simulated plant populations. - The American Naturalist 158(4): 438-450.

[25] Xu, S., Yu, Z., Zhang, K., et al. (2018): Simulating canopy conductance of the, Haloxylon ammodendron, shrubland in an arid inland river basin of northwest China. Agricultural and Forest Meteorology 249: 22-34.

[26] Yoda, K. (1963): Self-thinning in overcrowded pure stands under cultivated and natural conditions (Intraspecific competition among higher plants XI). - J. Biol. Osaka City Univ. 14: 107-129.

[27] Zhang, H., Wang, G. X., Shen, Z. X., et al. (2006): Effect of sensitivity to abscisic acid on scaling relationships for biomass production rates and body size in Arabidopsis thaliana. - Acta Physiologiae Plantarum 28(4): 373-379.

[28] Zhang, H., Wang, G., Zheng, K., et al. (2010): Mass-density relationship changes along salinity gradient in Suaeda salsa L. - Acta Physiologiae Plantarum 32(6): 1031-1037.

[29] Zhang, R. C., Lin, Y., Yue, M., et al. (2012): Effects of ultraviolet-B irradiance on intraspecific competition and facilitation of plants: self-thinning, size inequality, and phenotypic plasticity. - PLoS One 7(11): e50822.

[30] Zhang, W. P., Jia, X., Bai, Y. Y., et al. (2011): The difference between above-and belowground self-thinning lines in forest communities. - Ecological Research 26(4): 819-825. 
[31] Zhang, W. P., Jia, X., Wang, G. X. (2017): Facilitation among plants can accelerate density-dependent mortality and steepen self-thinning lines in stressful environments. Oikos. https://doi.org/10.1111/oik.03983.

[32] Zhang, Y., Xie, J. B., Li, Y. (2016): Effects of increasing root carbon investment on the mortality and resprouting of, Haloxylon ammodendron, seedlings under drought. - Plant Biology. https://doi.org/10.1111/plb.12511.

[33] Zou, T., Li, Y., Xu, H., et al. (2010): Responses to precipitation treatment for Haloxylon ammodendron growing on contrasting textured soils. - Ecological Research 25(1): 185194. 